\title{
Weltbilder und Weltordnung: Einführung in die Theorie der Internationalen Beziehungen
}

\author{
Gert Krell / Peter Schlotter \\ Baden-Baden 2018: Nomos, 5. überarbeitete und aktualisierte Auflage, 461 Seiten.
}

\section{Franz Eder}

University of Innsbruck, Department of Political Science, Austria

E-Mail: franz.eder@uibk.ac.at
Wer nach einem Einführungsbuch in die Internationalen Beziehungen (IB) sucht, der hat die Qual der Wahl. Neben den englischsprachigen Standardwerken wie The Globalization of World Politics (Baylis/Smith/Owens), International Relations Theory (Dunne/Kurki/Smith), International Relations (Grieco/Ikenberry/Mastanduno) oder Introduction to International Relations (Jackson/Sørensen), finden sich auch deutschsprachige Einführungen, die den Vergleich nicht scheuen müssen. Gerade sie können damit überzeugen, neben den klassischen Theorien auch die eher europäisch geprägten Entwicklungen in der Theoriebildung abzubilden. So finden sich in englischsprachigen Einführungen nicht immer Beiträge zur deutschen Regimeforschung, Englischen Schule oder gar marxistischen Ansätzen.

Unter diesen deutschsprachigen Einführungswerken sticht neben Lehrbuch der Internationalen Beziehungen (Deitelhoff/Zürn), Theorien der Internationalen Beziehungen (Schieder/Spindler - mittlerweile auch auf Englisch erschienen) und Internationale Politik (Schimmelfennig) vor allem Weltbilder und Weltordnungen von Gert Krell und Peter Schlotter heraus. Allein der Umstand, dass dieses Buch mittlerweile in der fünften Auflage erschienen ist, lässt erkennen, warum es zum Standardrepertoire der deutschsprachigen IB-Lehre gehört.

Von ursprünglich knapp 300 Seiten, ist Weltbilder und Weltordnungen mittlerweile auf 46I Seiten angewachsen und „globaler in seiner Perspektive geworden und stärker politisch-ökonomisch ausgerichtet" (7). Gerade darin liegt auch eine der großen Stärken dieses Buches. Krell und Schlotter betten die Theorien der IB gekonnt in den Kontext einer sich stark und rasch verändernde Welt ein und versuchen die Perspektive bewusst auch durch
Ansätze jenseits des angel-sächsisch und westeuropäisch geprägten „Mainstream“ zu erweitern.

Dabei sind die dreizehn inhaltlichen Kapitel alle nach dem gleichen didaktischen Schema aufgebaut: jedes Kapitel beginnt mit einem als „Lesehilfe“ bezeichneten Abstract und fasst mit „Was man weiß oder wissen sollte" die Kernpunkte der Darlegungen zusammen. Abschließend werden noch mögliche Diskussionspunkte für die Lehre und ausgewählte Literaturtipps zum Kapitelthema vorgeschlagen.

Das Buch selber gliedert sich in zwei Teile. Im ersten Teil, den sogenannten Voraussetzungen, legen Krell und Schlotter zunächst den Kontext dar, vor dessen Hintergrund sich die Theorien der IB entwickelt haben. Sie beginnen mit einer Einordnung der IB in das Fach Politikwissenschaft und zeigen, welche Rolle die Globalisierung für die Theoriebildung in den IB hat. Darauf aufbauend diskutieren sie die Entstehung der Moderne, des Kapitalismus, die Auswirkungen der Industriellen Revolution und Entwicklungen wie Kolonialismus, Imperialismus und Europas Rolle in diesen Prozessen. Die darauffolgende Auseinandersetzung mit dem Staat und dem Staatensystem ist genauso essentiell für das Verständnis internationaler Entwicklungen, wie die Einführung in das Völkerrecht, das den ersten Teil des Buches abschließt.

Diese Kontextualisierung ist die zweite große Stärke des Buches. Es gelingt den Autoren auf beeindruckende Weise mit einer zwar teilweise durchaus anspruchsvollen, aber auch für Laien nachvollziehbaren Sprache, den Leser in die Welt der IB zu entführen und sie für den zweiten Teil vorzubereiten.

In diesem zweiten Teil setzen sich die Autoren zum Ziel, durch eine Auseinandersetzung mit den Weltbil- 
dern (also den großen Denktraditionen) in den IB ein Verständnis dafür zu gewinnen, wie Menschen Ordnung in diese Welt der internationalen Beziehungen zu bringen versuchen. Sie beginnen dabei fast schon klassisch mit Thukydides, beschreiben ihn aber nicht wie üblich als Urvater des Realismus, sondern diskutieren sein Werk als ein vielschichtiges, indem Elemente nahezu aller großen Theorietraditionen zu finden sind.

Diese Theorietraditionen werden in den folgenden Kapiteln in all ihrer Breite diskutiert: vom Realismus, Liberalismus und Institutionalismus bis hin zu marxistischen und feministischen Ansätzen, sowie dem Konstruktivismus. Krell und Schlotter ergänzen diese Denkschulen im vorletzten Kapitel noch um politischpsychologische Theorien, also um Theorien mittlerer Reichweite. Die Vielfalt der von den Autoren präsentierten Ansätze reicht daher vom klassischen Realismus nach Morgenthau bis hin zu zeitgenössischen Debatten um soziale und ökologische Fragen auf globaler Ebene, sowie postkoloniale Deutungen um Konflikte und deren Ursachen.

Diese enorme Breite ist zugleich aber auch der Schwachpunkt dieses Werkes. Im Gegensatz zu Schieder/Spindler, die sich hauptsächliche auf das Werk von Referenztheoretikern und deren Kritik konzentrieren, lassen Krell und Schlotter eine Vielzahl an Theorien und deren Vertretern zu Wort kommen. Damit verlieren sie sich in der Unzahl an theoretischen Verästelungen, was zwangsläufig auf Kosten der theoretischen Durchdringung geht.

Der Leser wird im letzten Kapitel mit einer Diskussion der IB „zwischen Pluralisierung und Globalisierung“ aber wieder versöhnt. Aus einer humanistischen Perspektive kommen Krell und Schlotter nach der Diskussion postkolonialer Kritik am „westlichen“ Mainstream in den IB zum Schluss, dass Theorien der IB und die von ihnen gebildeten Kategorien „universale Gültigkeit beanspruchen“ (430) müssen, egal wo sie zum ersten $\mathrm{Mal}$ gedacht wurden. Damit reden sie einem pragmatischen Theorienpluralismus das Wort und stellen sich bewusst gegen den orthodoxen Glauben an die eine wahre Denktradition. Allein diese Erkenntnis wäre es schon Wert, dass sich Studierende mit den 46I Seiten von Weltbildern und Weltordnung ausgiebig auseinandersetzen.

\section{Literatur}

Baylis, John/Steve Smith/Patricia Owens (Hg.) (20I7), The Globalization of World Politics: An Introduction to International Relations, 7. Auflage, Oxford: Oxford University Press.

Deitelhoff, Nicole/Michael Zürn (2016), Lehrbuch der Internationalen Beziehungen: Per Anhalter durch die IB-Galaxis, München: C.H. Beck.
Dunne, Tim/Milja Kurki/Steve Smith (2016), International Relations Theory: Discipline and Diversity, 4. Auflage, Oxford: Oxford University Press.

Grieco, Joseph/G. John Ikenberry/Michael Mastanduno (2019), Introduction to International Relations: Perspectives, Connections, and Enduring Questions, 2. Auflage, London: Palgrave.

Jackson, Robert/Georg Sørensen (2016), Introduction to International Relations: Theories and Approaches, 6 . Auflage, Oxfort: Oxford University Press.

Schieder, Siegfried/Manuela Spindler (2019), Theorien der Internationalen Beziehungen, 4. Auflage, Opladen: UTB.

Schimmelfennig, Frank (2017), Internationale Politik, 5. Auflage, Opladen, UTB. 